developing dementia and rapid cognitive decline, and DLB. ${ }^{12}$ Similar VH are also reported in CBS, which was originally observed in elderly and visually impaired people without psychiatric or neurological problems. However, a close resemblance between CBS and PD related $\mathrm{VH}$ is also suggested. ${ }^{1}$

Recent neuropathological and radiological studies revealed that VH in CBS are associated with hyperperfusion of the temporal cortex, striatum, and thalamus, as well as increased cerebral activation in the ventral extrastriatal region, while DLB patients with VH showed hypoperfusion or hypometabolism of the occipital cortex. ${ }^{1}$ It was also reported that PD patients with VH also have alterations in or decreased cerebral activation in the temporo-parietal, parietal, and occipital regions. ${ }^{1}$ Our patient showed atrophy of the bilateral parieto-occipital, occipital, and mild left temporal lobes and decreased cerebral perfusion in the parietal and frontal lobes, indicating that the $\mathrm{VH}$ in our case may be caused by dysfunction of these regions. Such VH are rarely experienced by normal people, but, once seen, some people may be convinced they are supernatural events. We neurologists pay attention to such reports, and note the similarity in content between ghost tales and $\mathrm{VH}$, and pursue their mechanism of origin.

In conclusion, we propose that some ghost sightings are actually VH in normal people, which occur very rarely but more frequently than we in the medical establishment think.

\section{Electronic-database information}

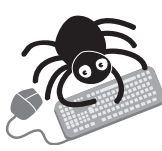

A longer version of the story by Shinya Nishimaru together with other ghost stories can be found in the supplemental information available at http://www. jnnp.com/supplemental.

H Furuya, K lkezoe Department of Neurology, Neuro-Muscular Centre, National Omuta Hospital, Fukuoka 837-0911, Japan

Y Ohyagi

Department of Neurology, Neurological Institute, Graduate School of Medical Sciences, Kyushu University, Fukuoka 812-8582, Japan

T Miyoshi

Department of Neurology, Omuta Rosai Hospital, Fukuoka 837-0904, Japan

N Fujii

Department of Neurology, Neuro-Muscular Center, National Omuta Hospital, Fukuoka 837-0911, Japan

Correspondence to: Dr H Furuya, Department of Neurology, National Omuta Hospital, Fukuoka 837 0911, Japan; furuya@oomuta.hosp.go.jp

Patient details are published with consent The ghost stories are reproduced with permission doi: 10.1136/jnnp.2004.055939

Competing interests: none declared

\section{References}

1 Diederich NJ, Goetz CG, Stebbins GT. Repeated visual hallucinations in Parkinson's disease as disturbed external/internal perceptions: focused review and a new integrative model. Mov Disord 2005;20:130-40.

2 Frucht SJ, Bernsohn L. Visual hallucinations in PD. Neurology 2002;59:1965.
3 Nishimaru S. Yama da, Genshijin da, Yuh-rei da [Mountaineering, primitive peoples and ghosts] (in Japanese). Kadokawa-Bunko. Tokyo, Japan: Kadokawa-Shoten, 1981:258-75.

4 Yanagida K. Toh-no Monogatari ['Toh-no' folklore] (in Japanese). Shincho-Bunko. Tokyo, Japan: Shincho-sya, 1977:51-139.

5 Tang-Wai DF, Graff-Radford NR, Boeve BF, et al. Clinical, genetic, and neuropathologic characteristics of posterior cortical atrophy. Neurology 2004;63:1168-74.

\section{Glucose metabolism and} dopamine PET correlates in a patient with myotonic dystrophy type 2 and parkinsonism

We describe a 72 year old woman who presented with a 5 year history of progressive gait uncertainty, frequent sudden falls, and difficulty rising from a chair, associated with fatigue. She noticed some degree of distal weakness in her arms when carrying weight compared with previous years. She had no complaints of pain or cramps but noticed stiffness and 'locked' legs when walking. She denied cranial nerve problems and had no general systemic complaints. Past medical history was significant for hypertension. Family history was unremarkable.

Examination revealed normal vital signs and intact cranial nerves. Slow and slightly slurred speech was evident. Her trunk was forcefully bent forwards in a camptocormic attitude, which could be corrected by passive extension of the trunk. Her tone was increased with rigid wrist and hips and brisk $(3+)$ reflexes and an equivocal bilateral extensor toe sign. Her gait was slow, and worsened during the day and over time to the extent that it limited everyday activities and often culminated in sudden falls. Pendular movements were reduced on the left. She had mild weakness in the shoulder abductors (4), finger flexors (4.5), hip flexors (4), and ankle dorsiflexors (4). Sensory examination and coordination were normal.

Routine blood investigation was unremarkable except for red blood cells $\left(3.84 \times 10^{6}\right)$, haemoglobin $(11.2 \mathrm{mg} / \mathrm{dl})$ and haematocrit $(34 \%)$ in the low normal range. Gammaglobulins were $7 \%$ of total protein levels. Electrocardiography revealed first degree arteriovenous block. Brain computed tomography scan demonstrated diffuse white matter vascular hypodensity and cortical atrophy. A diagnosis of parkinsonism was made. Treatment with Sinemet CR tablets (carbidopa/levodopa 25/100 mg twice daily) was started. However, despite treatment, the patient had not improved 5 months later. Muscle pain was present. Repeat examination showed severe neck flexor weakness (Medical Research Council scale <3).

The patient was unable to turn on her side in bed, or to lift her trunk from the bed when lying. Laboratory examination was unchanged except for a two fold increase in creatinine kinase (CK) levels. Needle electromyography (EMG) demonstrated diffuse myotonic discharges. Posterior iridescent initial cataract was demonstrated by slit lamp examination in the lens of the left eye. Fluorescent in situ hybridisation on muscle biopsy of the left biceps brachii demonstrated preferential nuclear clump type II fibre atrophy and ribonuclear inclusions, consistent with the diagnosis of myotonic dystrophy type 2 (DM2). (CTG) $)_{\mathrm{n}}$ expansion at the $D M P K$ gene was normal. The DM2 mutation was confirmed and diagnosis of DM2 was subsequently made.

Neuropsychological tests were administered as previously described. ${ }^{1}$ These included a screening test for dementia (Mini Mental State Examination) and tests of nonverbal reasoning (Raven's progressive coloured matrices), auditory language comprehension (Token test), verbal fluency with phonemic and semantic cues, verbal and spatial short term memory (Digit span forward and Spatial span), verbal and spatial long term memory (Story recall and Rey recall), constructional abilities (Rey's complex copy), attention and executive function (Trail Making A and B, Alertness and divided attention, Tower of London test, Wisconsin Card Sorting test). The patient showed an impairment of spatial long term memory (Rey recall: raw score 1 out of 36, cut off of normative data 9.97 out of 36) and of attention and executive function (Divided attention: raw score $892 \mathrm{~ms}$; cut off $811.7 \mathrm{~ms})$, and Tower of London test (percentage error $193 \%$, cut off $100 \%$ ). In the other tests, the patient's performances were normal.

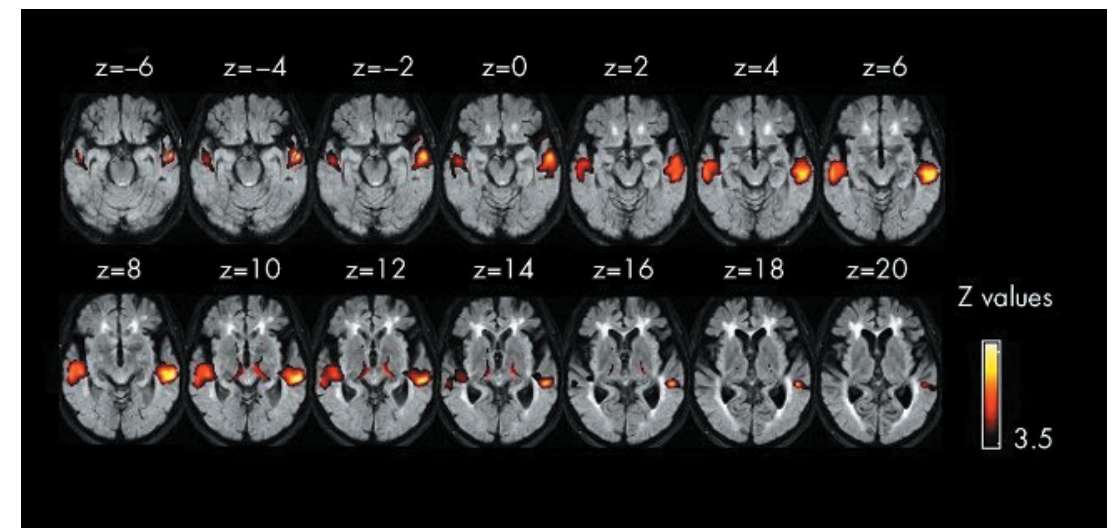

Figure 1 Statistical parametric maps of 18F-FDG PET showing significant metabolic reductions ( $p<0.05$ corrected) bilaterally in superior temporal gyrus and parietal operculum (Montreal Neurological Institute stereotactic coordinates of local maxima for suprathreshold clusters $x, y$, $z=64,-28,10, Z=6.01 ;-62,-22,6, Z=5.26)$, and thalamus $(18,-22,14, Z=5.06 ;-8$, $-16,12, Z=4.23)$, superimposed on the T2 weighted MRI of the patient. The areas indicated in shades of yellow and red both indicate areas of hypoperfusion; yellow represents areas of maximum hypoperfusion. 
Brain magnetic resonance imaging (MRI) demonstrated diffuse white matter lesions consistent with chronic hypoperfusion. ${ }^{1}$ The patient then underwent positron emission tomography (PET) studies with different tracers: ${ }^{18}$ F-FDG PET showed a reduction of glucose metabolism bilaterally in the superior temporal gyrus, parietal operculum, and, noteworthy, in the posterior thalamus (fig 1). The presynaptic dopamine reuptake as measured by ${ }^{11} \mathrm{C}-\beta$-CIT-FE ${ }^{2}$ and the postsynaptic D2 receptor density as measured by ${ }^{11} \mathrm{C}$-raclopride ${ }^{3}$ were both within the normal range values, thus revealing the integrity of the nigrostriatal pathway.

\section{DISCUSSION}

Our results confirm initial and more recent reports that extrapyramidal signs of different degrees may be present in DM2, ${ }^{25}$ yet emphasise that the diagnosis of DM2 is still often one of exclusion and is frequently unrecognised. Despite the growing number of reports describing the concomitant findings of myotonic dystrophy and extrapyramidal signs, the frequency and significance of this association is still unclear. Still less clear is the pathophysiology of extrapyramidal involvement and its therapeutic indications.

We describe a patient having camptocormia, bradykinesia, reduction of pendular movement and rigidity, suggesting features of Parkinson's disease (PD). This diagnosis was only subsequently excluded when there was no response to levodopa treatment, weakness and 'locking' worsened, and pain became a dominant symptom. Elevated CK and myotonic discharges led to the clinical diagnosis, subsequently confirmed by genetic analysis. Rigid spine syndrome was considered as a differential diagnosis because the bent spine attitude may result from weakness of the paraspinal and abdominal muscles, as in this patient. In addition, CK may be elevated and EMG may show spontaneous activity and a myopathic pattern. However, inflammatory signs on muscle biopsy are prominent in focal myositis, while other extrapyramidal signs such as those present in our patient are usually absent.

Although only carried out in a single patient, our PET studies demonstrate that parkinsonian features in DM2 are not related to neurodegenerative processes of the nigrostriatal system, such as in PD. Instead, they may be related to hypometabolism in the posterior thalamus. Studies on a larger group of patients with DM2 having extrapyramidal signs are needed to confirm these preliminary findings demonstrating the integrity of the dopaminergic system, and the therapeutic and prognostic implications.
V Sansone, G Meola

Department of Neurology, University of Milan Policlinico San Donato, Milan, Italy

D Perani, F Fazio

Vita-Salute San Raffaele University, IRCCS H San Raffaele, IBFM-CNR, Milan, Italy

V Garibotto, F Fazio

University of Milano-Bicocca, Milan, Italy

M Cotelli

UO Alzheimer, IRCCS Fatebenefratelli San Giovann di Dio, Milan, Italy

M Cotelli

Centro di Scienza Cognitiva, Univeristy of Turin, Turin,

Italy

E Vitelli

Department of Neurology, Ospedali Lodi, Milan, Italy

Correspondence to: Dr G Meola, Professor and Chair of Neurology, University of Milan, Policlinico San Donato, Via Morandi 30, 20097 San Donato Milanese, Milan, Italy; giovanni.meola@unimi.ił

doi: 10.1136/jnnp.2005.078451

Competing interests: none

\section{REFERENCES}

1 Meola G, Sansone V, Perani D, et al. Disexecutive dysfunction and avoidant personality trait in myotonic dystrophy type 1 (DM1-1) and in proximal myotonic myopathy (PROMM-DM2). Neuromusc Disord 2003;13:813-21.

2 Chu K, Cho JW, Song EC, et al. A patient with proximal myotonic myopathy and parkinsonism. Can J Neurol Sci 2002;29:188-90.

3 Lucignani G, Gobbo C, Moresco RM, et al. The feasibility of statistical parametric mapping for the analysis of positron emission tomography studies using 11C-2- $\beta$-carbomethoxy-3- $\beta$-(4-

fluorophenyl)-tropane in subjects with movement disorders. Nucl Med Commun 2002;23: 1047-55.

4 Volontè MA, Moresco RM, Gobbo C, et al. A PET study with [11-C]raclopride in Parkinson's disease: preliminary results on the effect of amantadine on the dopaminergic system. Neurol Sci $2001 ; 22: 107-8$.

5 Wunderlich S, Cxsoti I, Reioners K et al. Camptocormia in Parkinson's disease mimicked by focal myositis of the paraspinal muscles. Movement Disord 2002;17:598-600.

\section{BOOK REVIEW}

\section{Dementia with Lewy Bodies and Parkinson's disease dementia}

Edited by J O'Brien, I McKeith, D Ames, E Chiu. Published by Taylor \& Francis, 2005, London, $£ 75.00$ (€109; \$131 (hardcover)), pp 264. ISBN 1-84184-395-4
Dementia with Lewy Bodies (DLB) is a rare disorder? It was, but not anymore.. Following the publication of diagnostic criteria for DLB in 1996, research on this disorder witnessed a true explosion and the number of papers published each year increased by ten-folds. The wealth of knowledge that has been accumulating over the past 10 years on different aspects of DLB is now compiled in an easy-to-read volume. The book is systematically designed-an insightful first chapter on historical aspects is followed by a general overview on clinical spectrum of conditions which share Lewy bodies as their common pathological denominator and their classification. Authoritative sections on various aspects of the disease pursue including epidemiology, individual sections on cognitive, behavioural, somatic neurological and autonomic features, as well as the natural course of the disease, findings in neuro-imaging, underlying pathology and neurochemical deficits, and pharmacological and non-pharmacological treatment approaches. Some chapters provide comparison to a closely related disorder, dementia associated with Parkinson's disease, which is at the end also covered in a chapter of its own, along with a chapter devoted to differentiation of DLB from other disorders that may cause dementia and parkinsonism. Most sections are exhaustive with extensive coverage of the literature. Especially sobering is a first-hand account of how the disease is experienced by a caregiver, which would help clinicians to understand the real difficulties and set management priorities.

It was timely that the large amount of data that has been produced in the past decade was put together in a single volume, especially so as this has been largely missing. This is an excellent piece of work that will fill that gap, with chapters written by authors who have been in the forefront of research in this field. The timing is also fitting as revised diagnostic guidelines on DLB have just been published. This book will be a useful reading as well as an excellent source on the large amount of literature for those who have an interest in dementia, movement disorders and the border zone between them.

M Emre

\section{NOTICE OF RETRACTION}

doi: 10.1136/jnnp.2004.048363corr 1

Cittadini E, Goadsby PJ. Psychiatric side effects during methysergide treatment. J Neurol Neurosurg Psychiatry 2005;76:1037-8.

This paper has been withdrawn by the publisher. 